\title{
List of responses:
}

\section{For instance, the meaning of $I$ and $k$ in eq. (5) is still unexplained. The new sentence in lines $360-361$ is unclear. What is $c(5,95)$ ? The following paragraph is also confusing (probably due to a grammatical error).}

R: Definitions of I and k were extended in lines 262-277. The lines 360-365 were improved and explanation was extended. The definition of $c(5,95)$ was also considered in the paragraph

\section{I also, I would the authors to take into consideration the following points:} - When the intensity of the signal is negative, are the same values of $I$ and $k$ used? One would expect a different mechanism there, and therefore a different delay.

R: Regarding the combination of lags mentioned, we performed simulations with all possible combinations (with $k=1,2,3 ; I=1,2,3$, for $X$ and $Y$ respectively. Where $k=I=3$, the quantity of results within our sample with statistical significancy and were the ones presented in the paper. Information transference from both $\mathrm{X}->\mathrm{Y}$ and $\mathrm{Y}->\mathrm{X}(\mathrm{p}$-value $=<0.1)$ was the largest in any of the scenarios:

a) ETE $X->Y$ with $p$-value $=<0.10$ and ETE $Y$ - $>X$ with $p$-value $>0.10$, meaning that there is only information transfer from $X$ to $Y$.

b) ETE $X->Y$ with $p$-value $>0.10$ and ETE $Y->X$ with $p$-value $=<0.10$, meaning that there is only information transfer from $Y$ to $X$.

c) ETE $X->Y$ with $p$-value $=<0.10$ and ETE $Y->X$ with $p$-value $=<0.10$, meaning that there is information flux from both variables $X$ to $Y$ and viceversa.

To differentiate information flow, $X$ of $Y$, in Eq. (9), we defined how the intensity of the signal would be measured:

$$
\text { Intensity }=\operatorname{ETE}(Y \rightarrow X) /[\operatorname{ETE}(Y \rightarrow X)+\operatorname{ETE}(X \rightarrow Y)]
$$

In this equation, if the information signal was greater from $Y->X$, it would take a positive sign, meaning that there is a positive signal flowing from social media to stock prices, which is the main interest of our study. The contrary sign could be expected if the signal from $X->Y$ was greater. This was performed exclusively to differentiate the direction of the signal in our table results.

In addition, that the effect of the news in the stock market 3 days after the news is released is a result confirmed that well other authors shared in different papers quoted (Johan Bollen, Huina Mao, Xiaojun Zeng. Twitter mood predicts the stockmarket. Journal of Computational Science.2011; 2:1-

Tahir M. Nisar, Man Yeung. Twitter as a tool for forecasting stock marketmovements: A short-window event study. The Journal of Finance and DataScience 4.2018:101-119) 
- Fig. 6 does not show a clear-cut proof that negative signals have a stronger effect on the price than positive ones. This conclusion is therefore not sufficiently supported and should be weakened, or further analysis must be made.

Figure 6 was omitted for this review to avoid ambiguity in the presented results 